\title{
: \\ Tecnología, desarrollo y democracia: hacia otra artificialidad posible
}

\author{
Gustavo Giuliano
}

\begin{abstract}
电
RESUMEN

En este trabajo se pretende transmitir algunas cuestiones que se consideran claves para la reflexión actual de la tecnología. Estas son: (1) la presencia de elementos de análisis indecidibles; (2) la necesidad, en consecuencia, de una toma de posición; (3) la importancia, frente a la dominancia de los expertos, de la integración de la diversidad de actores sociales involucrados; (4) el carácter contra-hegemónico de la lucha por la reforma tecnológica; (5) la urgencia de la búsqueda de una racionalidad alternativa de base democrática. Por último se destaca la relevancia de la educación en tecnología desde los primeros años de escolarización y la importancia de la búsqueda de nuevos contextos epistémicos y políticos que permitan la participación responsable en el desarrollo tecnológico.
\end{abstract}

Palabras-cLave • Tecnología. Desarrollo. Democracia. Racionalidad.

\section{INTRODUCGIÓN}

Día tras día nos encontramos como sociedad y como individuos frente a nuevos y viejos desafíos ocasionados por las nuevas y viejas tecnologías que comparten nuestra realidad cotidiana y moldean, en mayor o menor medida, para mejor o para peor, nuestras formas de vida. Frente a este paradojal hecho, de aparición tardía, pero con una actividad intelectual intensa, se despliegan en el panorama académico actual diversos marcos teóricos que estudian las dinámicas involucradas en los procesos tecnológicos e indagan sobre la propia esencia de la actividad y la artificialidad que ésta construye.

Como es lógico de esperar de una disciplina joven que se interroga sobre un tema complejo, coexisten miradas que entienden y abordan al tema en estudio de maneras disímiles, incluso antagónicas e irreconciliables entre sí. En una aproximación gruesa, el filósofo de la tecnología canadiense Andrew Feenberg reconoce cuatro marcos teóricos que surgen de la combinación de dos elementos de análisis usualmente presentes: la modalidad del desarrollo y la caracterización moral de la tecnología 
(cf. Feenberg, 1999, p. 9). Esta propuesta de clasificación se resume en una tabla de doble entrada que determina cuatro posturas posibles, como se muestra en la figura $1 .{ }^{1}$

\begin{tabular}{|c|c|c|}
\hline La tecnología es: & Autónoma & Controlada \\
\hline Neutral & Determinismo & Instrumentalismo \\
\hline Valor dependiente & Substantivismo & Teoría crítica \\
\hline
\end{tabular}

Figura 1. Los cuatro marcos teóricos enumerados por Andrew Feenberg.

De la observación de la tabla, entrando por las filas, se alinean dos posibles valoraciones morales de la tecnología: quienes creen que es un proceso neutral en el que medios y fines son independientes, y los que, por el contrario, sostienen que medios y fines se influyen y definen mutuamente. Entrando por las columnas, se sitúan dos concepciones relativas al progreso: los que por un lado argumentan que el desarrollo tecnológico tiene características autónomas y los que, por el otro, defienden que el proceso es susceptible de ser social y políticamente controlado.

Los que apoyan la neutralidad y la autonomía - determinismo - asumen que la tecnología sigue una línea de progreso auto-referenciada que determina "natural e inevitablemente" (de aquí la neutralidad) el camino del progreso social y material. Argumentan que no se deben poner trabas a la innovación ya que si ocurren accidentes o consecuencias no previstas éstas son contingentes y serán resueltas con más y mejor tecnología, de acuerdo con las características propias de un proceso evolutivo.

La concepción que propone que la tecnología ya no puede concebirse como moralmente indiferente pero que admite, resignada, su automatismo - substantivismo está representada por gran parte de la tradición humanística, siendo el teólogo francés Jacques Ellul su principal referente. Para los adherentes a esta mirada, la tecnología no es neutral ya que sesga las posibilidades sociales de acuerdo a un programa que se ha vuelto autónomo, sin control humano, y que conlleva serios aspectos negativos de peligrosidad para la sociedad: contrariamente a sus promesas de libertad la tecnología terminará vaciando y esclavizando la vida del hombre.

1 Esta tabla se puede ampliar a más dimensiones, considerando, por ejemplo, cómo cada mirada entiende la relación entre ciencia y tecnología. 
Para los que defienden la neutralidad pero que admiten la posibilidad de control - instrumentalismo - la tecnología no es ni buena, ni mala en sí misma. Su carácter positivo o negativo, desde un punto de vista moral, depende de cómo se empleen los conocimientos, las técnicas y los artefactos que ella hace posible: la tecnología es sólo un medio neutral para obtener fines determinados por un agente exterior. Las valoraciones morales sólo surgen ante la elección de los fines a perseguir, ya que son éstos los que pueden ser buenos o malos. Esta mirada no atribuye relación causal unívoca entre tecnología y sociedad, pudiendo una misma tecnología ser compatible tanto con un sistema político de base capitalista liberal como con un socialismo planificado. Los que asumen esta posición insisten en la importancia de trabajar sobre el marco normativo, sobre la esfera simbólicamente mediada habermasiana.

Por último, se encuentran los que enmarcan a la tecnología en sí misma dentro del terreno de lo político - teoría crítica - y afirman que ya en la etapa de diseño se ponen en juego valores que condicionan el uso futuro por lo que se debe ejercer algún tipo de control en la etapa de modelado inicial. Negar que esto sea viable implicaría negar el ejercicio de la política y el pensamiento crítico. Sostienen que como agentes sociales productores de tecnología tiene que ser posible defender una posición que deje de adorar la maximización de la eficiencia técnico-económica como principal criterio de diseño y permita dar un rol más preponderante a otros valores, abriendo así el paso a una racionalidad tecnológica alternativa a la dominante.

\section{DesarRollo: haGia UNA TOMA DE POSicióN}

Ante este panorama diverso surge la primera de las cuestiones que se plantean aquí como relevantes para el interesado en reflexionar sobre los procesos tecnológicos: ¿cómo decidir dónde ubicarse? Uno de los pensadores actuales que aborda este interrogante es el filósofo francés Lucien Sfez para quien las posiciones y argumentaciones de los autores que se reconocen en cada vertiente están basadas muchas veces en afirmaciones que no son más que peticiones de principio, que parten, por ejemplo, desde definiciones que admiten ser puestas en discusión acerca de lo que se entiende por tecnología ${ }^{2}$ y que generan pendientes resbaladizas entre los diferentes marcos teóricos. Se constata así la presencia de una gran cantidad de enunciados indecidibles, en el sentido de irrefutables, que revelan el lugar fundamental que la ideología ocupa en la

2 Por enunciar sólo dos casos típicos, algunos autores piensan a la tecnología como sinónimo de artefactos técnicos mientras que otros lo hacen como ciencia aplicada. 
reflexión tecnológica y en la tecnología en sí misma (Sfez, 2005, [2002], p. 259-67). ${ }^{3}$ Esta característica, presente también en otros campos, tiene, en el que nos ocupa, efectos importantes en la acción derivados de las particularidades del objeto en estudio en tanto generador de artificialidad, ya que las conclusiones que se alcanzan no son meramente enunciativas sino que tienen consecuencias instituyentes de peso. Si la realidad no es algo inmutable predefinido de antemano sino que se construye socialmente de a poco, entonces los relatos que se proponen van permeando en la opinión pública de los ciudadanos y los gobernantes generando un imaginario que se plasma en actitudes concretas que lo auto-legitiman. En palabras de Sfez, "la ficción de la tecnología hace posible su realidad" (Sfez, 2005 [2002], p. 42). ${ }^{4}$

Este hecho conduce a la segunda de las cuestiones que entendemos centrales. Coincidiendo con Sfez, y referenciando nuevamente al cuadro de Feenberg, es claro que pensar la tecnología como autónoma y neutral no tiene igual potencial transformador sobre la sociedad que el hacerlo como controlada y valor dependiente. Ante una realidad social y medioambiental en muchos casos alarmante y con perspectivas complicadas, ${ }^{5}$ la singularidad del proceso tecnológico en cuanto a su capacidad formadora de sobre-naturalezas fuerza en consecuencia a una toma de posición, instituye un imperativo del orden de la urgencia. Se propone aquí que este imperativo debe exhortar a un diálogo de integración entre la totalidad de los actores sociales involucrados, que desdibuje los límites estancos entre quienes piensan y quienes hacen, expandiendo y re-significando las prácticas específicas sectoriales.

En este contexto que combina la falta de certezas epistémicas con la necesidad de actuar sobre la dinámica ciencia-tecnología-sociedad, brindar alternativas al modelo de desarrollo actual de bases científico-tecnológicas viables al menos en el mediano término, conlleva la incómoda necesidad de pensar y ejecutar formas de acción enraizadas en una comprensión aún inacabada del tema en estudio. Se trata, como sostiene el politólogo norteamericano Langdom Winner, de lo imperioso de forzar un "despertar del sonambulismo" que nos permita ser sujetos responsables aptos para transitar el presente y definir nuestro futuro (Winner, 1987 [1986], p. 26). No es fácil marchar en esta dirección frente a un fortísimo internalismo que pretende encerrar la ciencia dentro de los laboratorios, el proceso de diseño tecnológico dentro de las empresas y las políticas de desarrollo dentro de los gabinetes de gobierno, entes aislados habitados por sus respectivos "expertos".

3 Existe también para este autor una categoría de conceptos decidibles (refutables) como el de sistema técnico, linaje, salient reverse, acoplamineto y coeveolución entre otros.

4. Sfez se refiere claramente a la construcción de una hegemonía.

5 Recordemos que según datos de la Organización de las Naciones Unidas 26.000 niños mueren diariamente en el mundo por causas evitables asociadas a la pobreza. 
Se llega así a la tercera de las cuestiones que nos interesa señalar en tanto que bajo esta compartimentación se imposibilita articular una pregunta de importancia central para un diseño que armonice la relación ciencia-tecnología-sociedad: ¿cuál es la utilidad, necesidad, potencialidad y consecuencias de las innovaciones pretendidas por los agentes productores de ciencia y tecnología desde la perspectiva del contexto social amplio al cual afectará su descubrimiento y aplicación? No es posible abordar hoy esta pregunta de manera integral, sino que se la formula y responde sólo desde una mirada particular interesada, generalmente relacionada con intereses económicos corporativos y grupos de poder.

Se sostiene aquí que una genuina "revolución tecnológica" sólo surgirá de explorar una nueva manera de ligar la producción de conocimiento, el desarrollo tecnológico y los deseos y necesidades legítimos de las personas, atendiendo al desarrollo de una vida digna y a la realización de intereses auténticos y sustentables de las comunidades involucradas, desplazando una lógica de la innovación sustentada en el confort individual y el consumo por otra sostenida sobre la noción de bien común y solidaridad generacional e intergeneracional.

Si afirmamos, citando nuevamente a Andrew Feenberg, que se debe dejar de pensar a las técnicas como escindidas entre desarrollar y utilizar, ya que no son simples medios para las actividades humanas a los que se les pueden dar un buen o mal uso, sino que son fuerzas que moldean y condicionan a la sociedad, concluiremos con él que lo que aparentan ser meras elecciones instrumentales son en realidad elecciones acerca de la forma de la vida social y política que construye una sociedad. Sólo será posible entonces alcanzar un nuevo orden social si entendemos a la tecnología como perteneciente a la res publica, un asunto sobre el que no sólo se tiene el derecho a ser consultado sino también el deber de estar informado. Se debe marchar hacia una noción de racionalización fundada en la responsabilidad de la acción técnica - su diseño, implementación y uso - por los contextos humanos y naturales, en oposición a la hegemonía dominante, donde sólo tienen voz los "expertos" y los ciudadanos somos relegados, en el mejor de los casos, al papel de consumidores acríticos.

Se arriba de este modo a la cuarta de las cuestiones que nos importa resaltar. Se trata de la necesidad de pensar y articular una práctica contra-hegemónica sobre la base de que el desarrollo tecnológico no es unilineal, sino que se ramifica en muchas direcciones pudiendo alcanzar altos niveles a lo largo de más de una vía diferente, y sobre la premisa de que está sobredeterminado por una conjunción de factores a la vez contingentes (políticos) y estructurales (técnicos). 


\section{CierRe: EN BUSGA DE UNA SALIDA DEMOGRÁTICA}

Pararse en un lugar, asumir una toma de posición inicial a pesar de las incertidumbres, no niega la rigurosidad metodológica sino que permite trabajar sobre la validación de las premisas de un marco conceptual a construir sobre una base teórica y empírica que no considere la realidad como un reservorio de indicadores percibidos como datos abstractos estáticos sino dentro de una dinámica transformadora de lo establecido factible de auto-validarse a través del éxito de la acción. No es casual que vuelva a surgir aquí una analogía tecnológica, en tanto el proceso mismo sugerido se encuentra inscripto, en cuanto acción humana, dentro de un marco a su vez técnico y político.

Hablar del éxito conlleva la necesidad de analizar la posibilidad del fracaso siempre latente ante la incertidumbre asociada a la complejidad y lo desconocido. Para ello es fundamental desligar este concepto del de equivocación. Esto lleva a la quinta y última de las cuestiones que enumeramos al comienzo: ¿cómo se legitima la acción? Se afirma aquí que el camino es la participación ciudadana responsable y libre en un contexto de democracia ampliada. ${ }^{6}$ De este modo se enfrenta a las sociedades a asumir el ejercicio de la responsabilidad en su grado máximo, no se las libera de culpas sino que por el contrario se las anuda a sus actos, sean estos loables o condenables. Se legitima así el nuevo concepto propuesto por Feenberg de "racionalización democrática" (Feenberg, 1999, p. 131-47), una racionalidad tecnológica que no excluye al sujeto, reemplazándolo por prolijas y neutrales matrices de costo-beneficio, sino que lo integra en su complejidad y en su diferencia, en sus mayorías y en sus minorías.

Para ejercitar esta legitimación de la acción tecnológica desde sus propias bases interrelacionadas de diseño y uso se deben tanto crear nuevas formas de participación plural como revisar y potenciar prácticas y mecanismos ya conocidos. Entre las nuevas, creemos que el "contexto de implicación” de Tula Molina? es un paso significativo a favor de la vida en comunidad en tanto permite abordar la discusión sobre valores cognitivos y sobre valores sociales de modo simultáneo y no como dos discusiones casi sin contacto entre sí. En este sentido constituye un nuevo y prometedor contexto epistémico-social para el análisis y deliberación sobre las prácticas científicas y tecnológicas, sus fines, límites y capacidades.

Entre las ya conocidas, a revisar y expandir, sin lugar a dudas, la educación tecnológica en todos los niveles ocupa un lugar irremplazable. Resulta de importancia vital la formación social en ciencia y tecnología, como así también en la práctica extendida

6 Sobrevuela a esta afirmación la conocida sentencia sobre la que se sustenta toda política democrática: "los pueblos libres nunca se equivocan".

₹ Ver Tula Molina en este volumen. 
de los procesos democráticos, desde los primeros años de escolarización. Los espacios de reflexión sobre la tecnología deben alcanzar también a los currículos de las disciplinas técnicas, como la ingeniería, incorporando miradas sobre la especificidad disciplinar que contribuyan a formar profesionales responsables y críticos acerca de sus capacidades.

Se trata, en definitiva, de marchar hacia una "artificialidad alternativa" que permita un mundo más inclusivo. Esta posibilidad, oculta tras el velo de la hegemonía actual, puede parecer ingenua o utópica. Sin embargo, a pesar de las dificultades del esfuerzo intelectual, técnico, político y colectivo necesario para alcanzarla, creemos que es el camino a seguir si queremos asumir con responsabilidad la construcción de un futuro digno, para nosotros y para quienes nos continúen.@

\title{
Gustavo Giuliano
}

Facultad de Ingeniería y Ciencias Físico-matemáticas, Universidad Católica Argentina, Buenos Aires.

Instituto de Estudios y Formación, Central de los Trabajadores Argentinos, Argentina. gustavo_giuliano@yahoo.com.ar

\begin{abstract}
This paper is meant to inform about some basic issues in connection with the current reflection on technology. These are: (i) the presence of elements which can not be decided; (ii) the necessity, in consequence, of assuming a position; (iii) the importance of integrating the diversity of social actors involved, facing the dominance of the experts in the issue; (iv) the anti-hegemonic character of the fight for technological reform; (v) the urge for a search of alternative rationality on a democratic basis. Finally, it highlights the relevance of teaching technology from the first years of formal education and the quest for new political and epistemic contexts which can allow responsible participation in technological development.
\end{abstract}

KeYwords • Technology. Development. Democracy. Rationality.

\section{REFERÊNGIAS BIBLIOGRÁFIGAS}

Feenberg, A. Questioning technology. New York: Routledge, 1999.

SFEz, L. Tecnología e ideología: Un juego de poder. Buenos Aires; Siglo xxI, 2005 [2002].

WinNer, L. La ballena y el reactor: una búsqueda de límites en la era de la alta tecnología. Barcelona: Gedisa, 1987 [1986]. 